\title{
One-dimensional upward continuation of the ground magnetic field disturbance using spherical elementary current systems
}

\author{
Heikki Vanhamäki, Olaf Amm, and Ari Viljanen \\ Finnish Meteorological Institute, Geophysical Research Division, P.O. Box 503, FIN-00101 Helsinki, Finland
}

(Received June 27, 2003; Revised October 28, 2003; Accepted November 20, 2003)

\begin{abstract}
Ionospheric equivalent currents are defined as spherical sheet currents, which reproduce the observed magnetic disturbances below the ionosphere. One way of determining these currents is to place several so called spherical elementary current systems (SECS) in the ionospheric height and to solve an inversion problem for the amplitudes of these systems. In previous studies this method has been applied to two-dimensional data sets, having both latitudinal and longitudinal spatial coverage (2D SECS method). In this paper a one-dimensional variant of this method (1D SECS) is developed. The 1D SECS method can be used even in those situations where the data set is one dimensional, e.g. with one meridionally aligned magnetometer chain. The applicability of the 1D SECS method is tested using both synthetic and real data. It is found that in real situations the errors in the 1D SECS results are $5-10 \%$ in current density profiles and $\sim 5 \%$ in integrated currents, when compared to the results of the more accurate 2D SECS method.
\end{abstract}

\section{Introduction}

The determination of ionospheric equivalent currents from measurements of ground magnetic field disturbances is of primary interest in ionosphere-magnetosphere research (e.g. Untiedt and Baumjohann, 1993). Several different techniques, like spherical or spherical cap harmonic expansions of the disturbance field (Haines, 1985), have been developed over the years. Amm and Viljanen (1999) introduced the spherical elementary current system (2D SECS) method, which is very flexible and well suited for local and global studies. Advantages of this method are that no global wavelength cut-offs are needed (as in harmonic expansions) and the resolution of the analysis grid (density of elementary systems) may vary from place to place according to the amount of available data. A one-dimensional variant of this method (1D SECS) is now presented.

In this context one-dimensionality means that the situation is independent of the azimuthal coordinate of the chosen spherical coordinate system. In this study we use geographical coordinate system (which we assume to be spherical) and the 1D SECS is derived so that several (infinitely many) 2D SECSs of identical amplitude are placed uniformly at a constant latitude at the ionospheric height. The resulting current system (1D SECS) varies only in latitude. The ground magnetic field of such a system is calculated and the ionospheric equivalent currents may be obtained by placing several 1D SECSs of different magnitude at different latitudes, so that their combined ground magnetic field matches the measured field disturbance as closely as possible. In this approach the effect of internal currents is assumed to be neglible. Alternatively the internal equivalent currents could be modelled

Copy right (C) The Society of Geomagnetism and Earth, Planetary and Space Sciences (SGEPSS); The Seismological Society of Japan; The Volcanological Society of Japan; The Geodetic Society of Japan; The Japanese Society for Planetary Sciences. by placing a similar 1D SECS system below the ground. In this way the ground disturbance field could be separated into internal and external parts (see Pulkkinen et al., 2003b, for 2D SECS case).

The 1D SECS method should work best in electrojet situations, when strong, mostly east-west aligned currents flow in the ionosphere, for these often are almost one-dimensional. In addition, the 1D SECS method can be used even if there is data from only one meridian magnetometer chain. In the latter case the original 2D SECS method may not work very well, for the regions just outside the magnetometer network may be badly resolved even when using data from two-dimensionally distributed network, see Pulkkinen et al. (2003a), and with one-dimensional chains this problem would be more severe. The applicability of the 1D SECS method is tested in different electrojet conditions using synthetic data. Also other 1D upward continuation methods have been developed over the years, e.g. 1D Fourier (Mersmann et al., 1979) and 1D current strip method (Popov et al., 2001), and in this study we compare the results of the 1D SECS and 1D Fourier methods. Real data is also used to investigate the applicability of the 1D methods to situations that in fact are weakly or strongly two-dimensional. The results of the 2D SECS method are used as references in these cases.

\section{2D SECS and Equivalent Currents}

Amm (1997) defined two different spherical elementary sheet current systems, one being divergence-free $\left(\mathbf{J}_{e l, d f}\right)$ and the other curl-free $\left(\mathbf{J}_{e l, c f}\right)$, see Fig. 1. The curl-free system is associated with field aligned currents (FACs) corresponding to $\nabla \cdot \mathbf{J}_{e l, c f}$ that are assumed to flow radially outwards or inwards. Written in a spherical coordinate system $\left(r^{\prime}, \theta^{\prime}, \phi^{\prime}\right)$, with unit vectors $\left(\hat{\mathbf{e}}_{r^{\prime}}, \hat{\mathbf{e}}_{\theta^{\prime}}, \hat{\mathbf{e}}_{\phi^{\prime}}\right)$, having its pole at the center of 


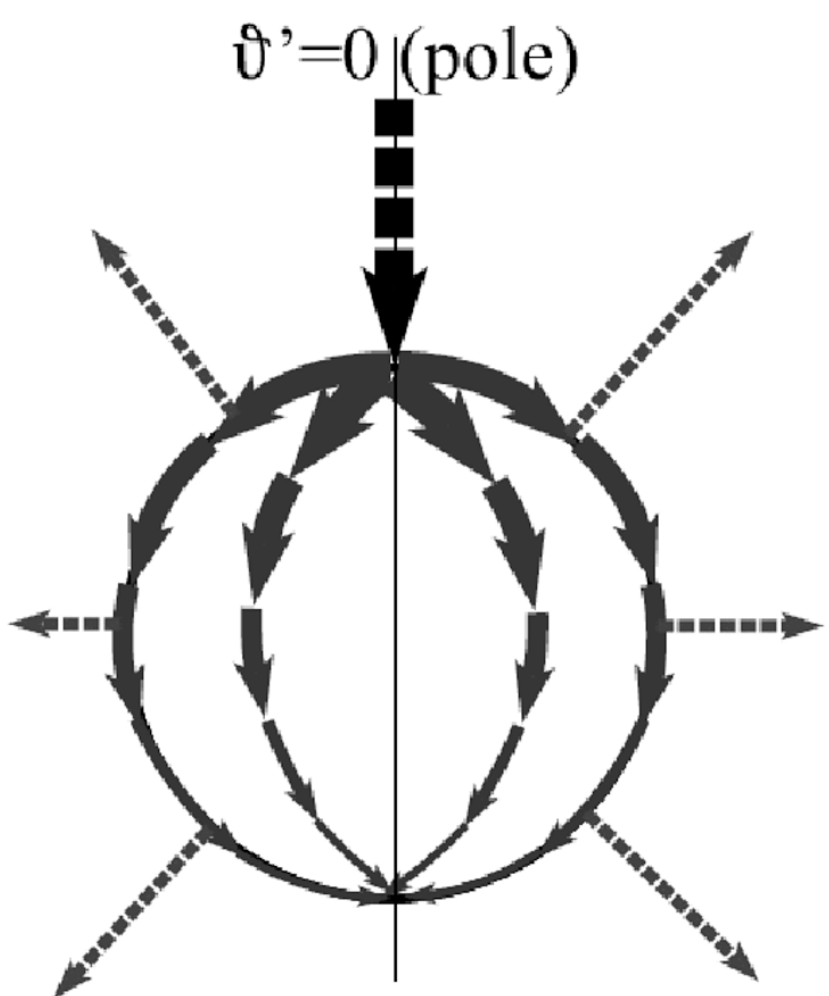

Curl-free elementary system (with associated FACs)

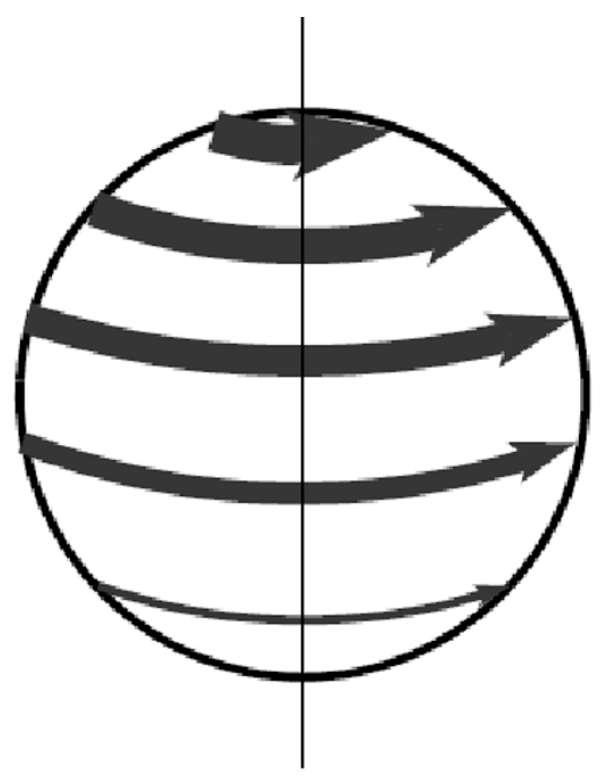

Divergence-free elementary system

Fig. 1. The divergence- and curl-free elementary current systems. Poles $\theta^{\prime}=0$ of these systems may be placed at different points in the ionosphere.

the elementary systems, the currents are

$$
\begin{aligned}
& \mathbf{J}_{e l, c f}\left(r^{\prime}, \theta^{\prime}\right)=\frac{I_{0, c f}}{4 \pi R_{I}} \delta\left(r^{\prime}-R_{I}\right) \cot \left(\frac{\theta^{\prime}}{2}\right) \hat{\mathbf{e}}_{\theta^{\prime}} \\
& \mathbf{J}_{e l, d f}\left(r^{\prime}, \theta^{\prime}\right)=\frac{I_{0, d f}}{4 \pi R_{I}} \delta\left(r^{\prime}-R_{I}\right) \cot \left(\frac{\theta^{\prime}}{2}\right) \hat{\mathbf{e}}_{\phi^{\prime}} .
\end{aligned}
$$

Here $I_{0,\left[\begin{array}{c}c f \\ d f\end{array}\right]}$ is the magnitude of the current system and $R_{I}$ is the radius of the ionosphere, assumed to be a thin layer $100 \mathrm{~km}$ above Earth's surface.

Amm (1997) also showed that any ionospheric horizontal current distribution may be uniquely constructed as a superposition of these elementary systems by placing their poles all over the ionosphere. In fact, the elementary systems defined above can be used to expand any continuosly differentiable vector field on a sphere.

The curl-free current system $\mathbf{J}_{e l, c f}$, with associated FACs, does not produce any magnetic field below the ionosphere, as can be shown using Ampere's law (Fukushima, 1976). Ionospheric equivalent currents, which are spherical sheet currents that produce the same magnetic effects below the ionosphere as the original 3D currents, can be selected to be divergence-free. Consequently the ground magnetic disturbance field may be reproduced by placing several $\mathbf{J}_{e l, d f}$ systems in the ionosphere above the area of interest and choosing their magnitudes appropriately. This is the 2D SECS method for determining ionospheric equivalent currents, developed by Amm and Viljanen (1999) and further validated by Pulkkinen et al. (2003a). In general, the ground magnetic disturbance is caused by both external (ionospheric) and internal (induced) currents and only the horizontal part of the measured disturbance field can be fitted using just ionospheric equivalent currents. Field separation into internal and external parts may be carried out by placing another equivalent current layer inside the ground and matching all components of the disturbance field (Pulkkinen et al., 2003b).

\section{The 1D SECS Method}

\subsection{Current distribution and magnetic field}

In the 2D SECS method poles of the current system $\mathbf{J}_{e l, d f}$ are placed at different positions $\left(\theta_{0}, \phi_{0}\right)$. The unprimed coordinate system $(r, \theta, \phi)$ with unit vectors $\left(\hat{\mathbf{e}}_{r}, \hat{\mathbf{e}}_{\theta}, \hat{\mathbf{e}}_{\phi}\right)$ refers to the geographic spherical coordinates, whereas the pole of the primed system is fixed to the 2D SECS system in question. The $1 \mathrm{D}$ variant of the SECS method is obtained by integrating over the longitude $\phi_{0}$ of the 2D SECS pole, so that the resulting current system is independent of longitude and depends only on latitudes $\theta$ of the observation point and $\theta_{0}$ of the poles.

The ionospheric current density of an 1D elementary system is calculated from the integral

$$
\mathbf{J}_{1 D}=\frac{I_{0}}{4 \pi R_{I}} \delta\left(r-R_{I}\right) \int_{0}^{2 \pi} \cot \left(\frac{\theta^{\prime}}{2}\right) \hat{\mathbf{e}}_{\phi^{\prime}} d \phi_{0}
$$

Using identities of spherical trigonometry the angle $\theta^{\prime}$ and unit vector $\hat{\mathbf{e}}_{\phi^{\prime}}$ can be expressed in terms of coordinates $\theta, \theta_{0}, \phi_{0}$ and unit vectors $\hat{\mathbf{e}}_{\theta}, \hat{\mathbf{e}}_{\phi}$. Some details of the calcu- 


\section{IMAGE Magnetometer Network}

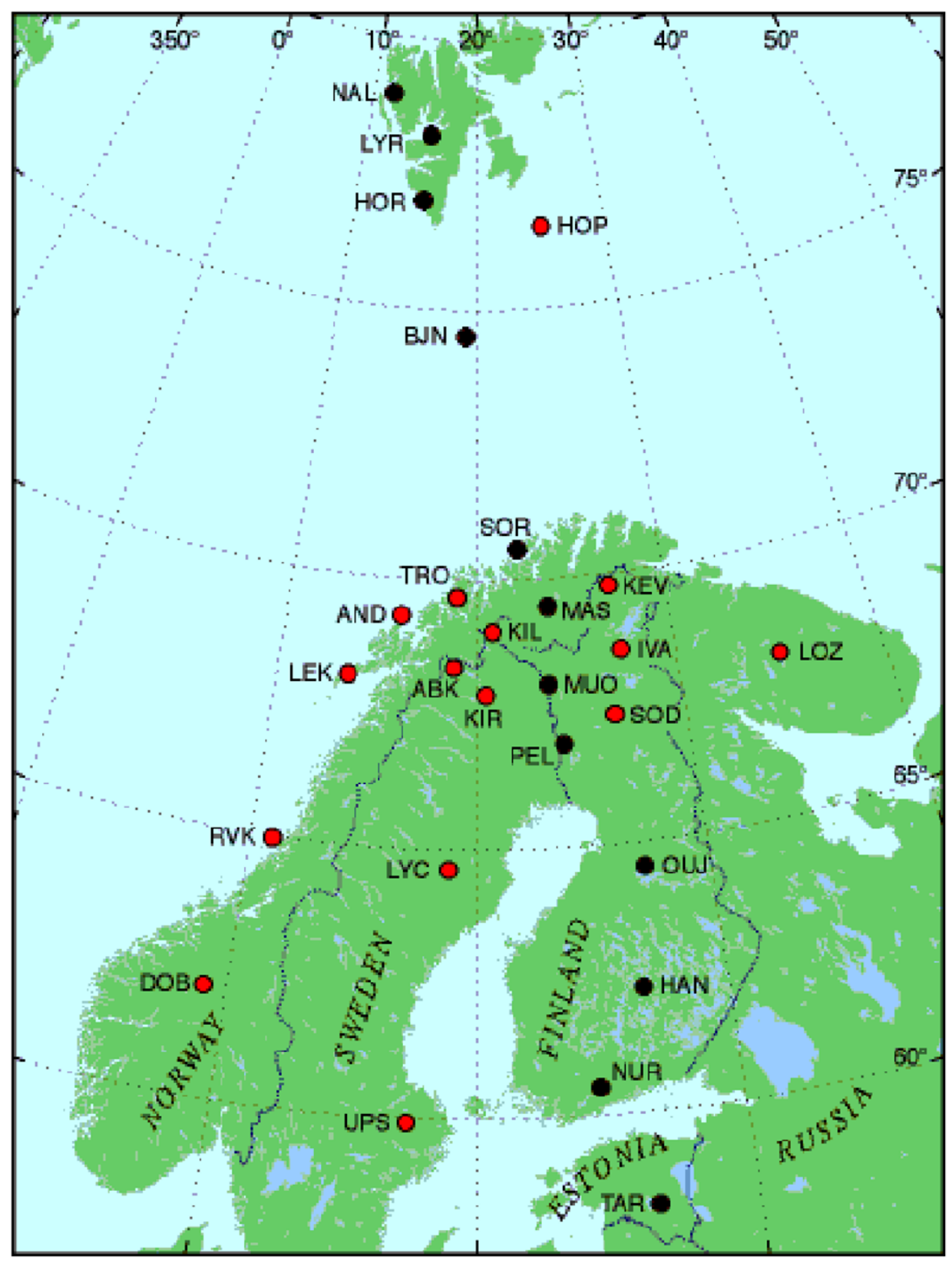

October 2001

Fig. 2. The north-south chain used in this study is marked in black.

lation are given in Appendix A. The result is

$$
\mathbf{J}_{1 D}\left(r, \theta, \theta_{0}\right)=\frac{I_{0}}{2 R_{I}} \delta\left(r-R_{I}\right) \hat{\mathbf{e}}_{\phi} \begin{cases}-\tan \left(\frac{\theta}{2}\right) & , \theta<\theta_{0} \\ \cot \left(\frac{\theta}{2}\right) & , \theta>\theta_{0}\end{cases}
$$

The magnetic field caused by the 1D SECS may be obtained by first calculating the vector potential

$$
\mathbf{A}(\mathbf{r})=\frac{\mu_{0}}{4 \pi} \int \frac{\mathbf{J}_{1 D}\left(r_{1}, \theta_{1}, \theta_{0}\right)}{\left|\mathbf{r}-\mathbf{r}_{1}\right|} d \mathbf{r}_{1}
$$

Integration may be carried out by expanding the denominator in spherical harmonics and the magnetic field is obtained as $\mathbf{B}=\nabla \times \mathbf{A}$. Details of the calculation are given in Appendix B.
The azimuthal component $B_{\phi}$ of the magnetic field is zero. The other components can be written using Legendre polynomials $P_{l}^{m}$ (with the Condon-Shortley phase convention) as

$$
\begin{gathered}
B_{r}=\frac{\mu_{0} I_{0}}{2 r} \sum_{l=1}^{\infty}\left(\frac{r}{R_{I}}\right)^{l} P_{l}\left(\cos \theta_{0}\right) P_{l}(\cos \theta) \\
B_{\theta}=\frac{\mu_{0} I_{0}}{2 r} \sum_{l=1}^{\infty}\left(\frac{r}{R_{I}}\right)^{l} \frac{1}{l} P_{l}\left(\cos \theta_{0}\right) P_{l}^{1}(\cos \theta) .
\end{gathered}
$$

These results are valid for $r<R_{I}$. Similar expressions for the case $r>R_{I}$, needed e.g. in modelling internal equivalent currents, are given in Appendix B. In numerical calculations 
A

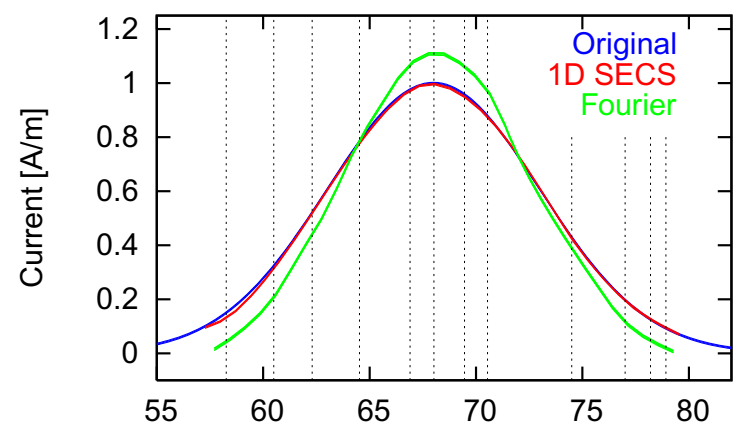

C

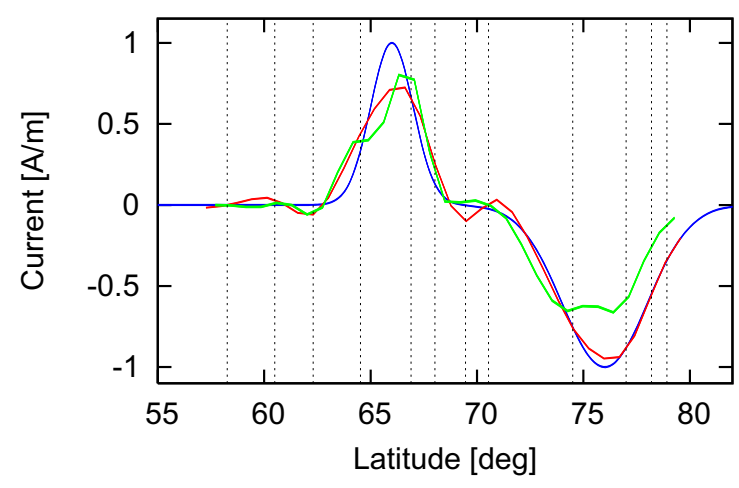

B

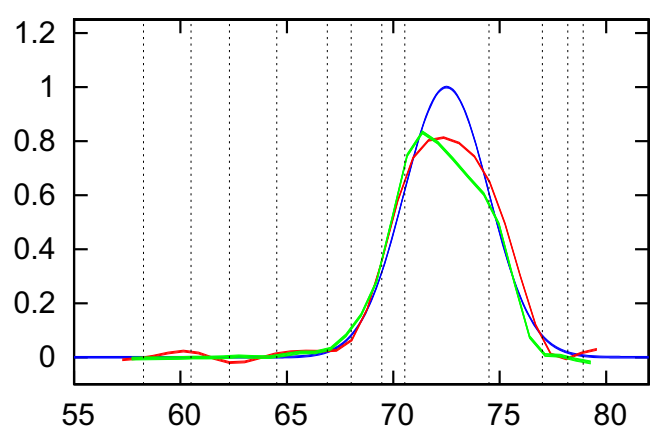

D

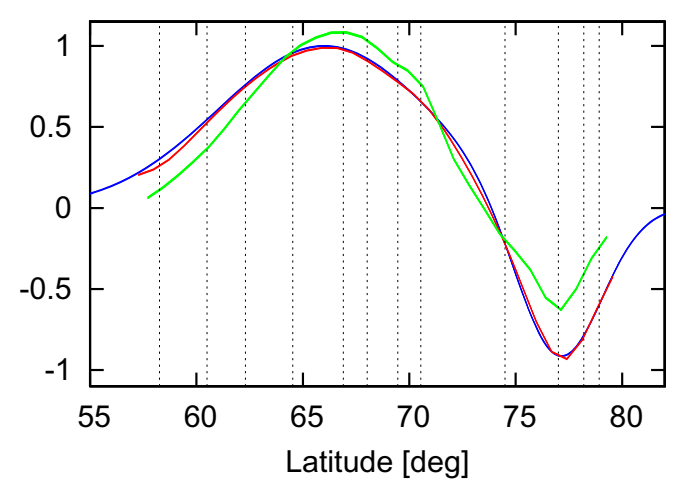

Fig. 3. Various original current distributions and those obtained using 1D SECS and Fourier methods. Magnetometer positions are marked with vertical lines.

of the series in Eqs. (6) and (7) several hundred terms (usually about 200) have to be evaluated to get good convergence. This does not affect the computational efficiency of the 1D SECS method, for the series have to be evaluated just once for a given magnetometer and 1D SECS configuration, as explained below.

\subsection{Calculation of equivalent currents}

The ionospheric equivalent currents are calculated so that several 1D SECSs are placed at different latitudes $\theta_{0, j}, j=$ $1 . . n$, and their magnitudes are chosen so that the north-component ( $x$-component) of their combined ground magnetic field matches the measured disturbance at latitudes $\theta_{i}, i=$ 1..m, as closely as possible. This may be expressed as a matrix equation

$$
\mathbf{B}_{x}=\overline{\bar{M}} \cdot \mathbf{I}_{0},
$$

where $\mathbf{B}_{x}, \mathbf{I}_{0}$ are vectors containing the observed $x$-components at $\theta_{i}$ and the amplitudes of the 1D SECSs at $\theta_{0, j}$, respectively, and $\overline{\bar{M}}$ is a matrix that gives the effect of a current system at $\theta_{0, j}$ to a magnetometer at $\theta_{i}$.

The matrix $\overline{\bar{M}}$ depends only on the geometry of the situation and it may be calculated using Eq. (7) once the latitudes of the magnetometers and 1D SECSs are specified. In practice the number of magnetometers $(m)$ is usually much smaller than the number of 1D SECSs $(n)$ placed in the ionosphere. As a rule of thumb the spacing of the 1D SECSs should be smaller than $1 / 2$ of the magnetometer spacing and the area where 1D SECSs are placed should exceed the area of the magnetometer network by few degrees. Some fine tuning is of course required for different magnetometer networks. Thus the inversion problem is highly underdetermined and special methods must be used to solve the unknown $\mathbf{I}_{0}$ in Eq. (8). One possibility is the singular value decomposition (SVD, e.g. Press et al., 1992, section 2.6).

In SVD the matrix $\overline{\bar{M}}$ is decomposed into $\overline{\bar{M}}=\overline{\bar{U}} \overline{\bar{w}} \overline{\bar{V}}^{T}$, where $\overline{\bar{U}}, \overline{\bar{V}}^{T}$ are orthogonal matrices and $\overline{\bar{w}}$ is a diagonal matrix. The diagonal elements $w_{k k}$ of $\overline{\bar{w}}$ are called the singular values of $\overline{\bar{M}}$ and $n-m$ of them are zeros due to the underdetermination of the problem. Also some of the nonzero singular values may be very small (compared to the largest) and they represent the badly behaving part of the solution. The solution is made well behaving by setting all $w_{k k}<\epsilon \cdot \max \left(\left|w_{k k}\right|\right)$ equal to zero. Here $\epsilon$ is an adjustable parameter (in practise $\epsilon=0.01 . .0 .1$ ). After this has been done the problem may be solved as

$$
\mathbf{I}_{0}=\overline{\bar{V}} \operatorname{diag}\left(\tilde{w}_{k k}\right) \overline{\bar{U}}^{T} \mathbf{B}_{x},
$$

where $\tilde{w}_{k k}=w_{k k}^{-1}$ if $w_{k k} \neq 0$, zero otherwise.

In the above method only the $B_{x}$-component is used as input. In principle, by including the internal equivalent currents, also the vertical $z$-component could be matched. The inversion problem is solved only for the amplitudes of the 1D SECSs. Because each elementary system has global current distribution, given by Eq. (4), there is no need to solve an inversion problem for the positions of the 1D SECSs, if they are chosen reasonably in the first place. It should also be mentioned that in general the unprimed coordinate system 


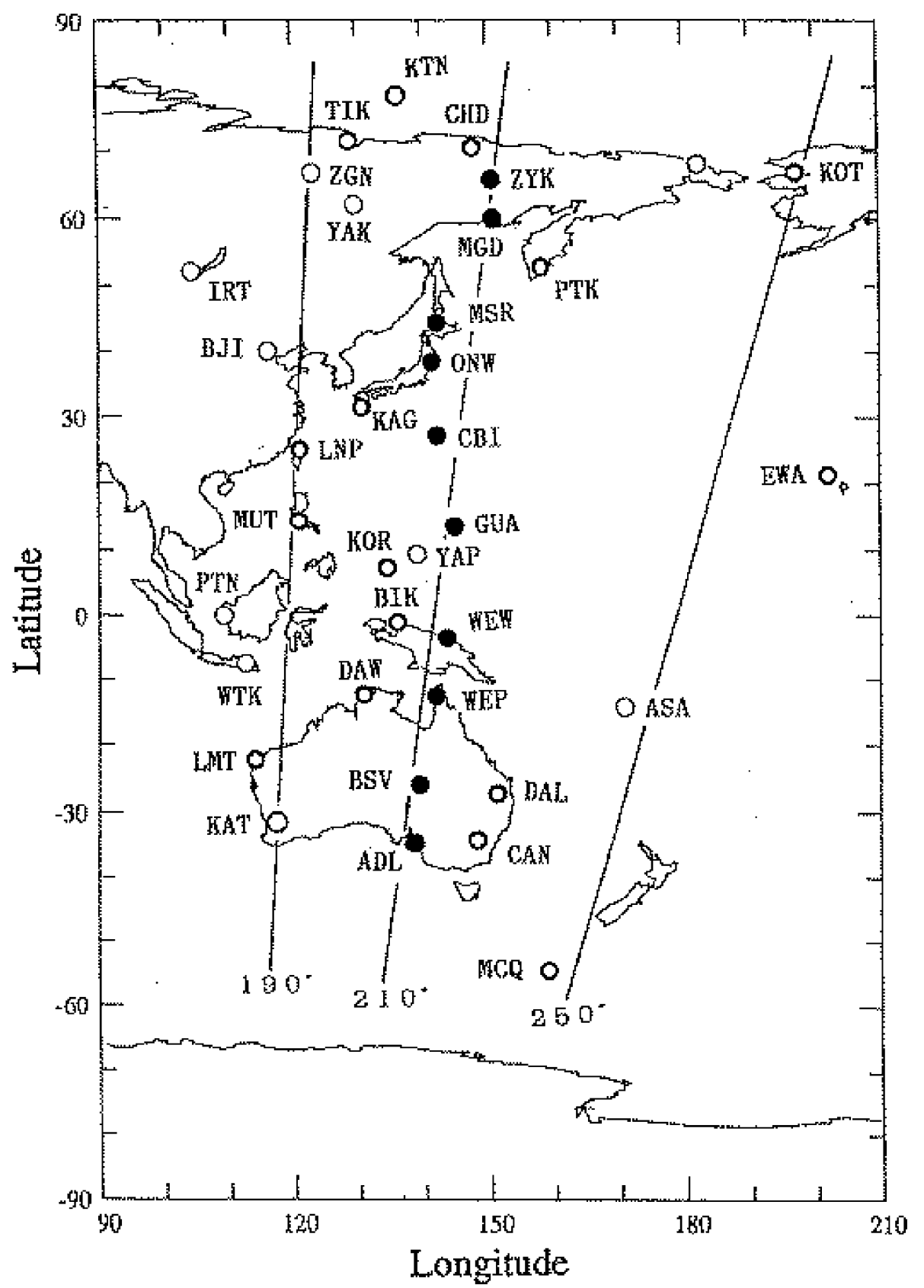

Fig. 4. Locations of the $190^{\circ}, 210^{\circ}$ and $250^{\circ}$ MM chain stations. The north-south chain used in this study is marked in black.

need not be the geographical system. Any other spherical coordinate system, e.g. geomagnetic one, could be used.

\section{Application Example-Simulated Data}

We selected one north-south chain of magnetometers from the IMAGE network ${ }^{1}$ as our observation points (see Fig. 2) and simulated magnetic disturbance data for these stations by placing different one-dimensional $\left(\mathbf{J}_{1 D}(\theta) \| \hat{\mathbf{e}}_{\phi}\right)$ current distributions in the ionosphere.

An electrojet having amplitude $A$, width $D$ and central latitude $L$ is modelled as

$$
\mathbf{J}_{1 D}(\theta)=A \exp \left[\frac{-(\theta-L)^{2}}{2 D^{2}}\right] \hat{\mathbf{e}}_{\phi} .
$$

Several electrojets may be superposed and their current distribution is expanded as a superposition of 1D SECSs (Eq. (4)) so that the ground magnetic field can be calculated easily. This expansion is in practise identical to the original current distribution, unless it contains step-functions or the like.

$B_{x}$ at the magnetometers is then used as input for the 1D SECS method and the output current distribution can be directly compared against the original one. We also compared the results of the 1D SECS method with those of the 1D Fourier method in Cartesian coordinate system (Mersmann et al., 1979).

A general description of the 1D Fourier method is: 1) The $x$-component of the field is expanded as a Fourier series. 2) In upward continuation to height $h$ the expansion coefficients 
27/08/1998 23:25:00

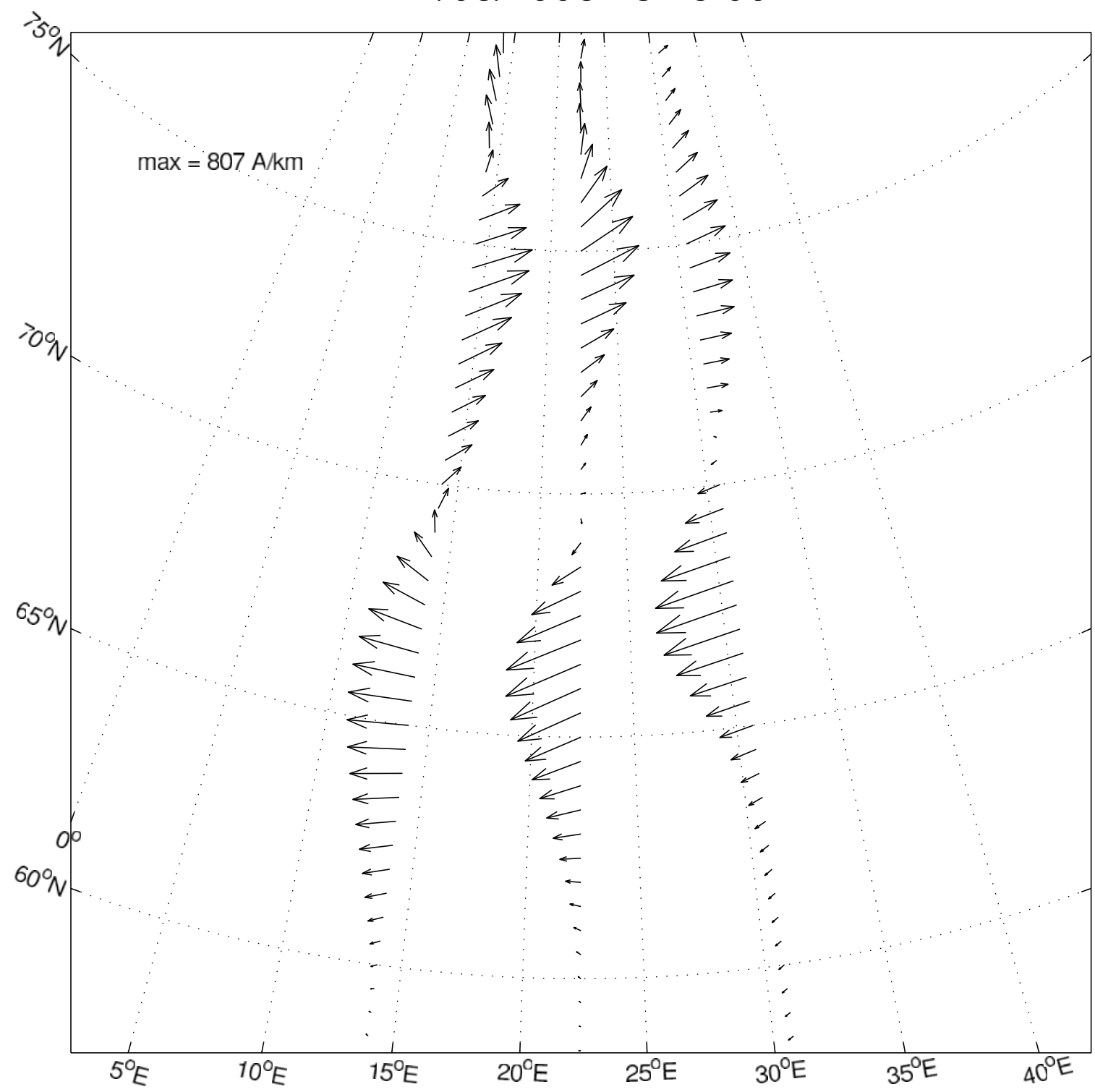

Fig. 5. Ionospheric equivalent currents along three meridians as given by the 2 D SECS method.

Table 1. The percentual errors of the 1D SECS and Fourier results for test cases A-D of Fig. 3, see Eq. (11).

\begin{tabular}{ccccc}
\hline & $\mathrm{A}$ & $\mathrm{B}$ & $\mathrm{C}$ & $\mathrm{D}$ \\
\hline 1D SECS & 0.5 & 3.8 & 5.6 & 1.9 \\
Fourier & 4.8 & 3.7 & 10.7 & 10.8 \\
\hline
\end{tabular}

of wave number $k$ scale as $e^{k h}$. 3) Inverse Fourier transform gives $B_{x, \text { cont }}$ 4) Equivalent currents, obtained using Ampere's law, are $J_{y, e q}=\frac{2}{\mu_{0}} B_{x, \text { cont }}$.

\subsection{Test results}

Examples of different test current distributions together with those obtained from the 1D SECS and Fourier methods are shown in Fig. 3. Positions of the twelve magnetometers are also indicated with vertical lines. In the 1D SECS method elementary systems were placed uniformly at $40 \mathrm{~km}\left(0.35^{\circ}\right)$ intervals in the ionosphere and the $\epsilon$ parameter was 0.015 .
The calculation area was extented $\sim 5^{\circ}$ outside the magnetometer chain. In the Fourier method wavelengths smaller than $80 \mathrm{~km}$ were ignored and the data was spline interpolated and windowed before the Fourier transform. All these parameters were varied to find a combination that would work well in all situations studied.

In general the results of the 1D SECS method are better than those of the Fourier method, see Table 1. In cases A and D of Fig. 3, where current distributions are relatively smooth and situated well over the IMAGE chain, the 1D SECS results are almost identical to the original distributions. The gap in the magnetometer chain between $70^{\circ}-75^{\circ}$ does not appear to affect the results in these cases. However, with sharper electrojets the spacing of the magnetometers begins to affect the accuracy of the results. This is illustrated in cases $\mathrm{B}$ and $\mathrm{C}$, where some deviations from the original distributions can be seen in both methods. Especially the Fourier method has difficulties with the current peaks and in the 1D SECS result there are some oscillations outside the 

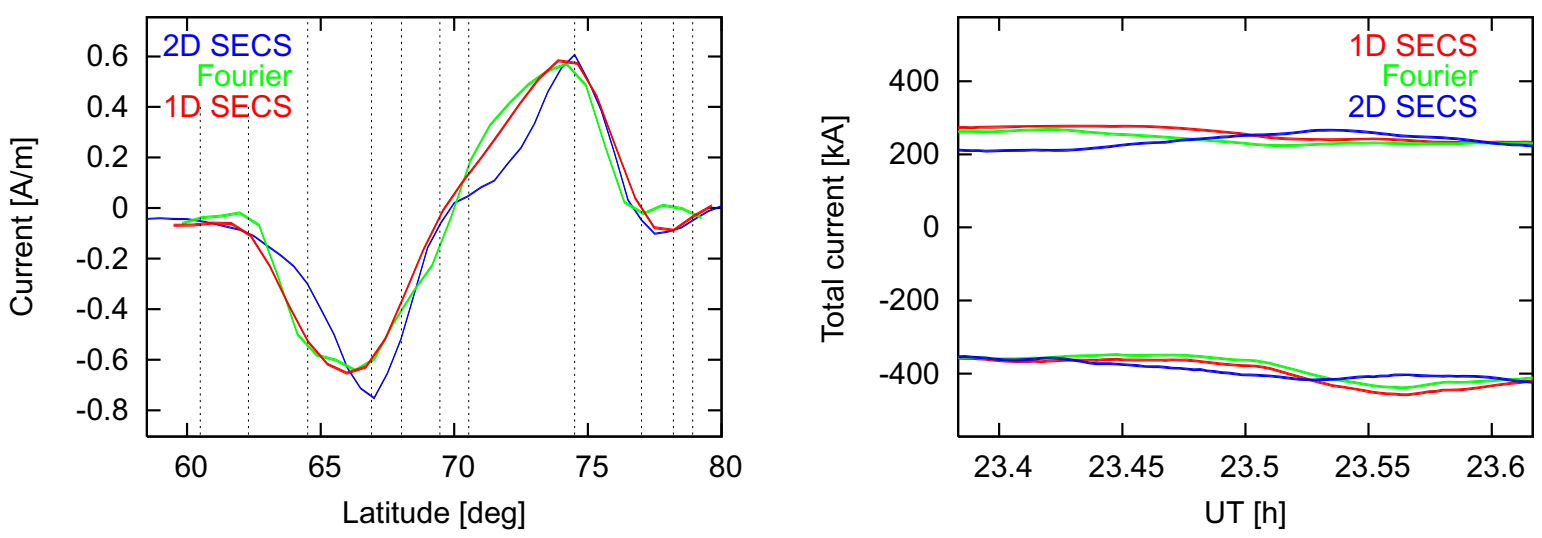

Fig. 6. LEFT: Snapshot of latitudinal current distribution over IMAGE at 23:25 UT on 27/8/1998 given by the 1D SECS, Fourier and 2D SECS methods. Eastward currents positive. RIGHT: Integrated eastward (+) and westward (-) currents corresponding to the same situation.

A $21: 31: 30$

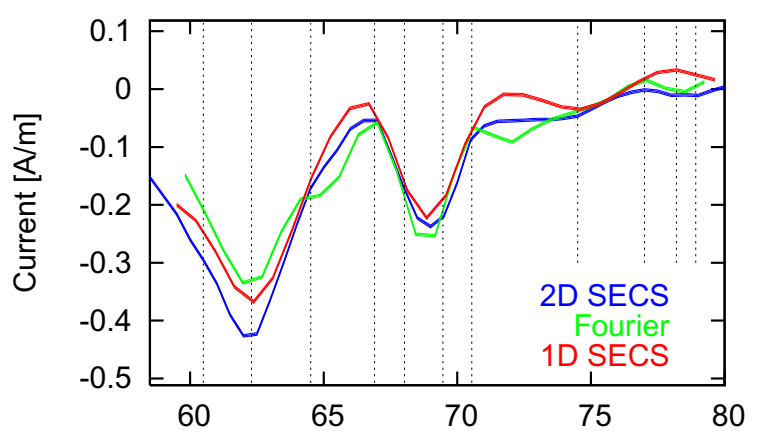

C $22: 30: 0$

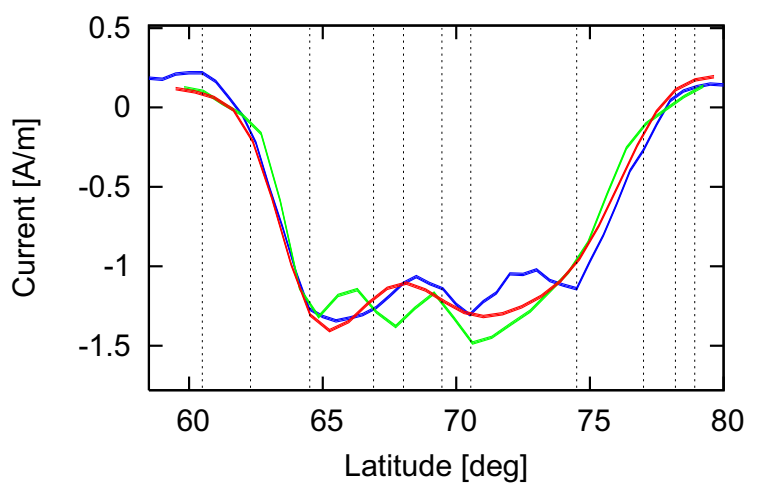

B 22:10:0

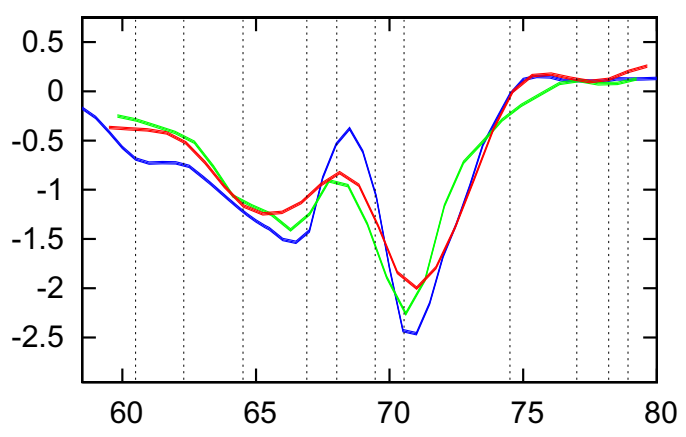

D 23:0:0

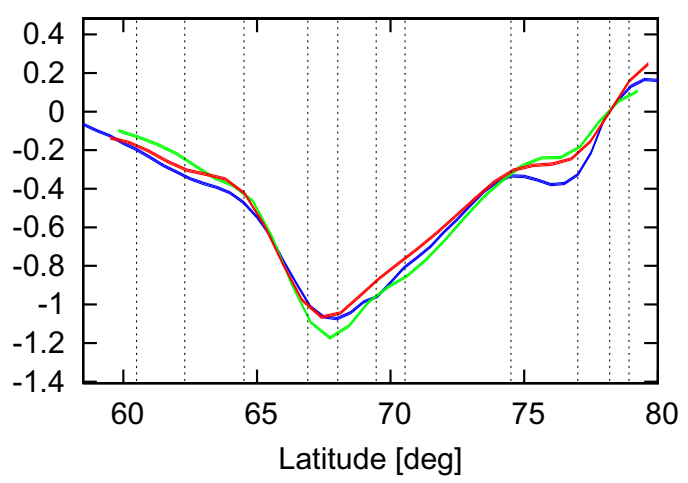

Fig. 7. Same as left panel of Fig. 6, but for 13/11/1998.

electrojets. Furthermore, the 1D SECS method seems to be able to reproduce the original currents over the magnetometer area better than the Fourier method in cases where large part of the currents are flowing outside the chain area (results not shown).

As a quantitave estimate for the performance of the $1 \mathrm{D}$ methods we calculated the absolute average error of their results, defined as

$$
\text { Error }=\left\langle\frac{\left|J_{1 D}-J_{\text {Orig }}\right|}{\max \left(\left|J_{\text {Orig }}\right|\right)}\right\rangle_{\text {lat }} .
$$

Here $<>_{\text {lat }}$ means average over latitude, $J_{1 D}$ is the $1 \mathrm{D}$ current density (1D SECS or Fourier) and $J_{\text {Orig }}$ is the original current density. Results for the four test cases shown in Fig. 3 are given in Table 1. These result can be compared directly, for $\max \left(\left|J_{\text {Orig }}\right|\right)=1$ Am in all test cases.

\section{Application Example-Real Data}

In order to test the 1D SECS method with real data we selected three different electrojet situations observed with IMAGE and one observed with the 210 Magnetic Meridian chain $^{2}$. The 2D SECS method was used as a reference in the IMAGE cases. The 1D methods used data from only one north-south chain of magnetometers, consisting of 11 


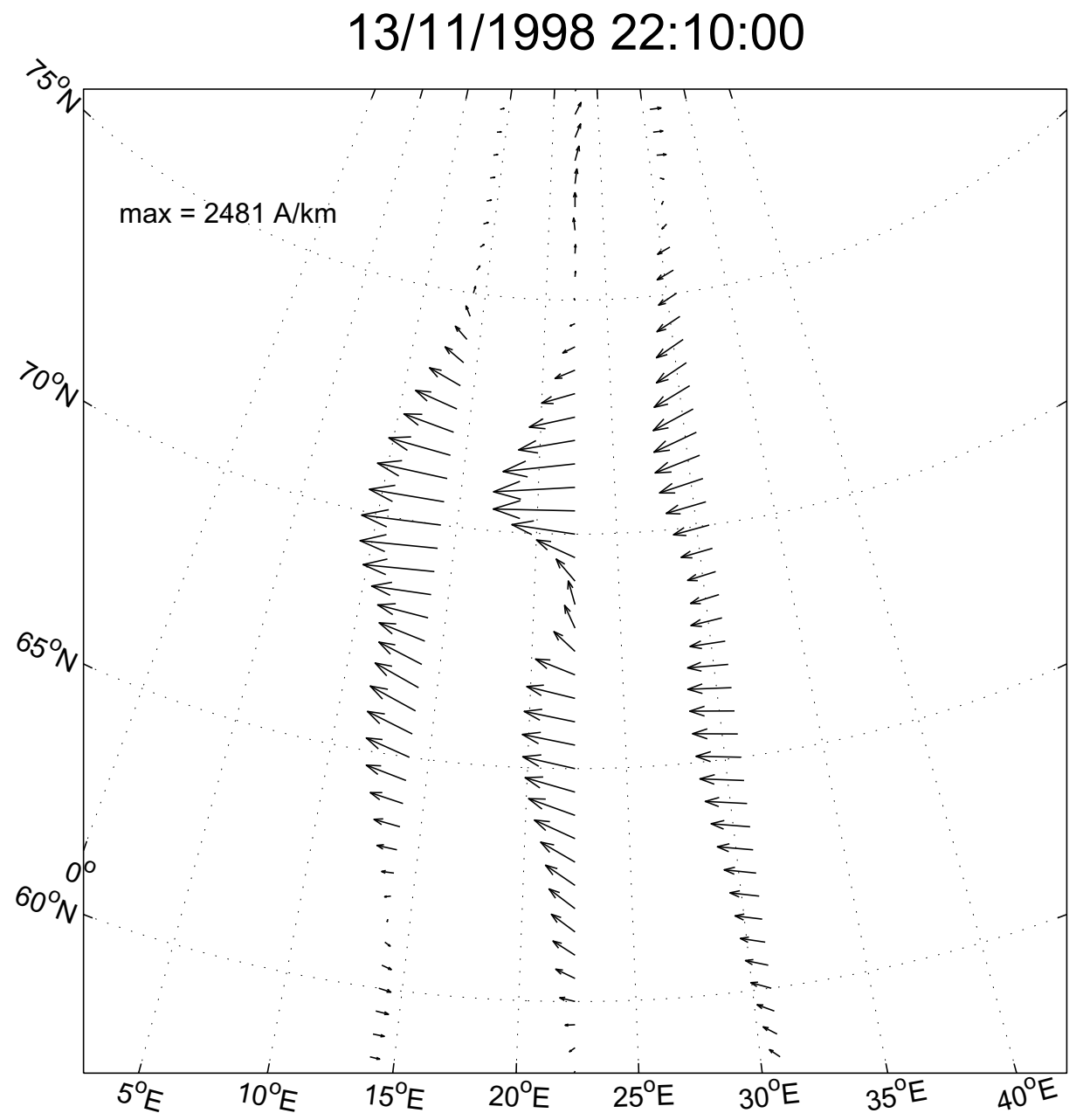

Fig. 8. Same as Fig 5, but for the time of panel B of Fig. 7.

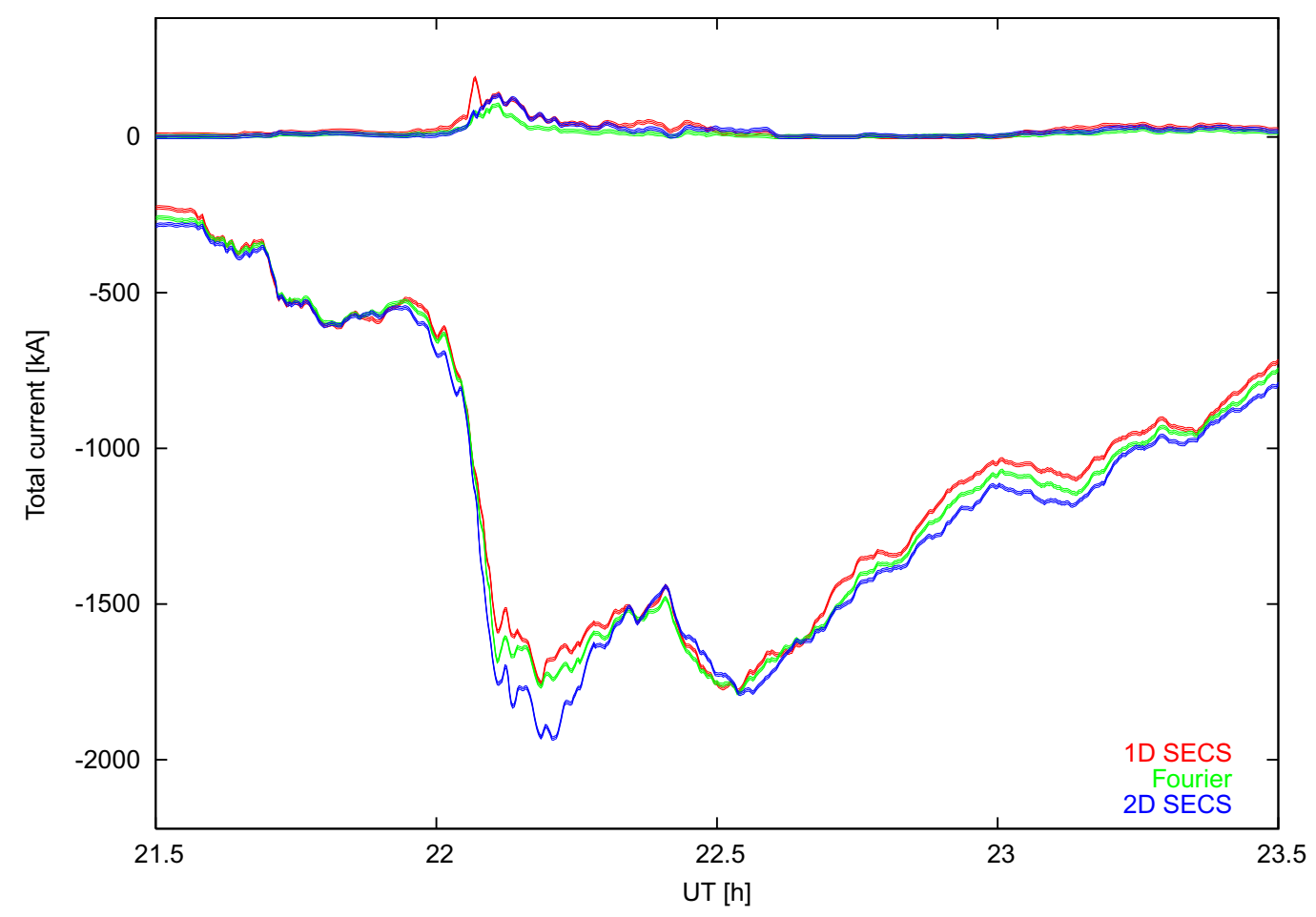

Fig. 9. Same as right panel of Fig. 6, but for 13/11/1998. 
A $5: 41: 30$

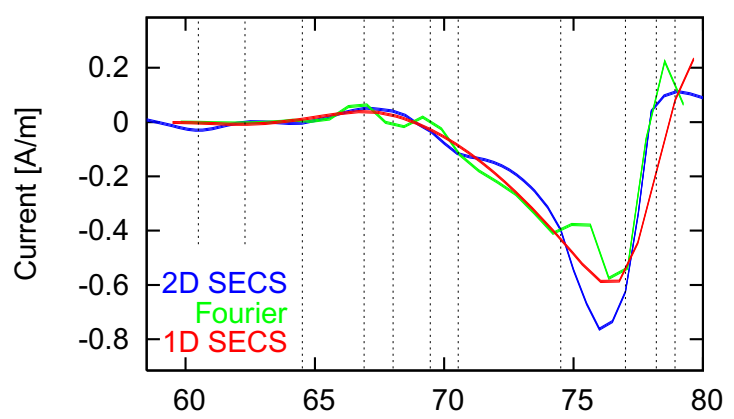

C $6: 43: 10$

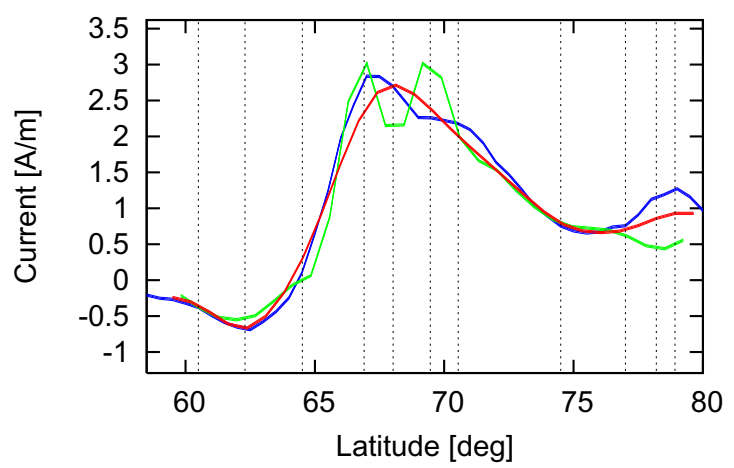

B $\quad 6: 13: 10$

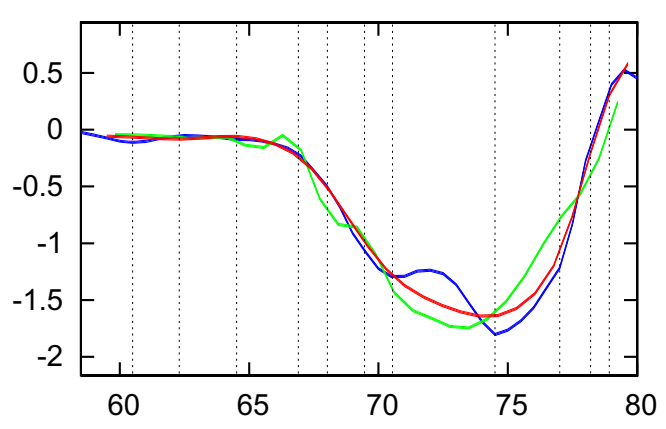

D $\quad 7: 49: 50$

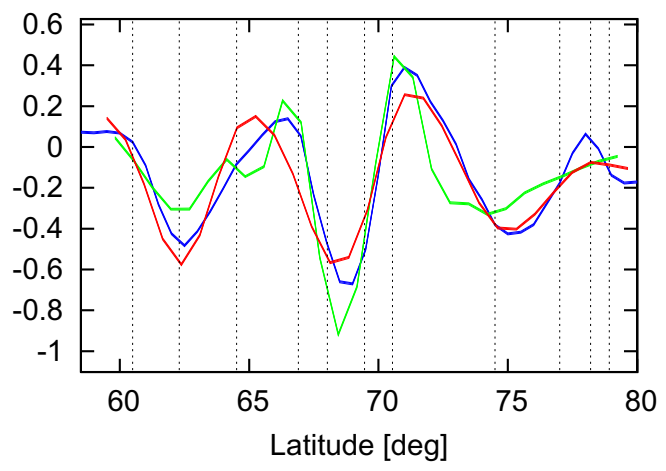

Fig. 10. Same as left panel of Fig. 6, but for 24/11/2001.

stations when using IMAGE data (Fig. 2, no data from TAR) and of 10 station with $210 \mathrm{MM}$ (Fig. 4). The 2D SECS method used data from all available IMAGE stations.

As the first example we studied a shear flow situation that occured close to UT midnight on 27/8/1998 over IMAGE. The situation was essentially stationary during the studied time period 23:23-23:37 UT and the two-dimensional equivalent currents obtained from the 2D SECS method are shown in Fig. 5. Both of the two electrojets are tilted from the east-west direction and they are not exactly one-dimensional, as their direction and magnitude change along their flow direction. The latitudinal distribution of east-west aligned equivalent currents crossing the $1 \mathrm{D}$ magnetometer chain at 23:25 UT, as given by the 1D SECS, Fourier and 2D SECS methods, is shown in Fig. 6. The total integrated eastward and westward currents for the studied time interval are also shown. The current distribution is similar to test cases $C$ and D of Fig. 3 and both 1D methods give similar results. The 2D SECS method gives slightly different current distribution and integrated currents. These differences are probably caused by the tilt and non-1D nature of the electojets.

The second example is a substorm that occured in the evening of 13/11/1998. The results of the different methods are shown in Figs. 7 and 9. The direction of the electrojet varied between southwest and northwest, as determined from the 2D SECS results, and most of the time the magnitude of the current varied with longitude. The greatest differences from one-dimensionality occured around 22:10 UT, see Fig. 8, but even then the 1D methods gave quite good results, as shown in panel B of Fig. 7. It appears that the nonone-dimensionality of the electrojet affects the 1D methods more than slight deviations from east-west direction. In the situation of panel D in Fig. 7, at 23:00 UT, the westward electrojet was almost $1 \mathrm{D}$ but tilted about $30^{\circ}$ from west towards south.

The third example is a local storm on $24 / 11 / 2001$ that began with a sudden storm commencement (SSC) just before 6 UT and lasted about 2 hours. Results for this case are shown in Figs. 10 and 11. As in the previous cases, the integrated currents given by different methods are very similar to each other, while the latitudinal current profiles show some differences. In general, the current systems were almost all the time $2 \mathrm{D}$ and tilted from the east-west direction, sometimes very much so. Situation was worst around 7:50 UT, when large part of the currents were flowing southwards and the current system was quite complex and variable. However, even in this case the 1D methods were able to reproduce the main characteristics of the east-west currents.

The errors of the 1D SECS and Fourier results in the above three examples are listed in Table 2. The errors in the current profiles were calculated as in Eq. (11) for each timestep, except that the 2D SECS results were used as reference. These errors, averaged over time, are given in the upper part of Table 2. In the lower part of Table 2 are the errors in the integrated currents for the three examples. These were calculated as

$$
\text { Error }=\left\langle\frac{\left|\left(J_{E, 1 D}^{\mathrm{int}}-J_{W, 1 D}^{\mathrm{int}}\right)-\left(J_{E, 2 D}^{\mathrm{int}}-J_{W, 2 D}^{\mathrm{int}}\right)\right|}{J_{E, 2 D}^{\mathrm{int}}-J_{W, 2 D}^{\mathrm{int}}}\right\rangle_{\text {time }}
$$

Here $E(W)$ means the positive (negative) integrated currents, $1 \mathrm{D}$ stands for the $1 \mathrm{D}$ SECS or Fourier methods and 


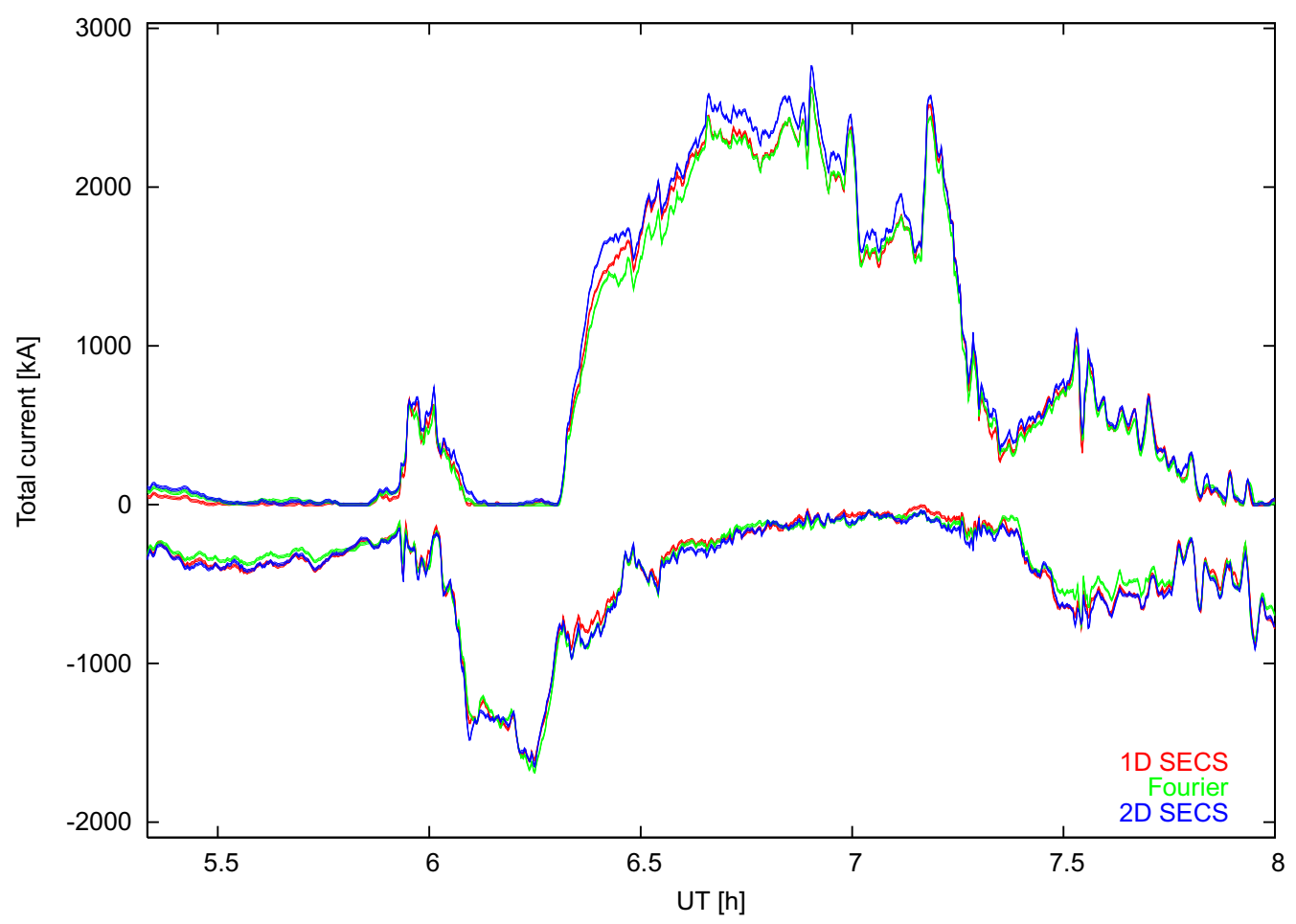

Fig. 11. Same as right panel of Fig. 6, but for 24/11/2001.
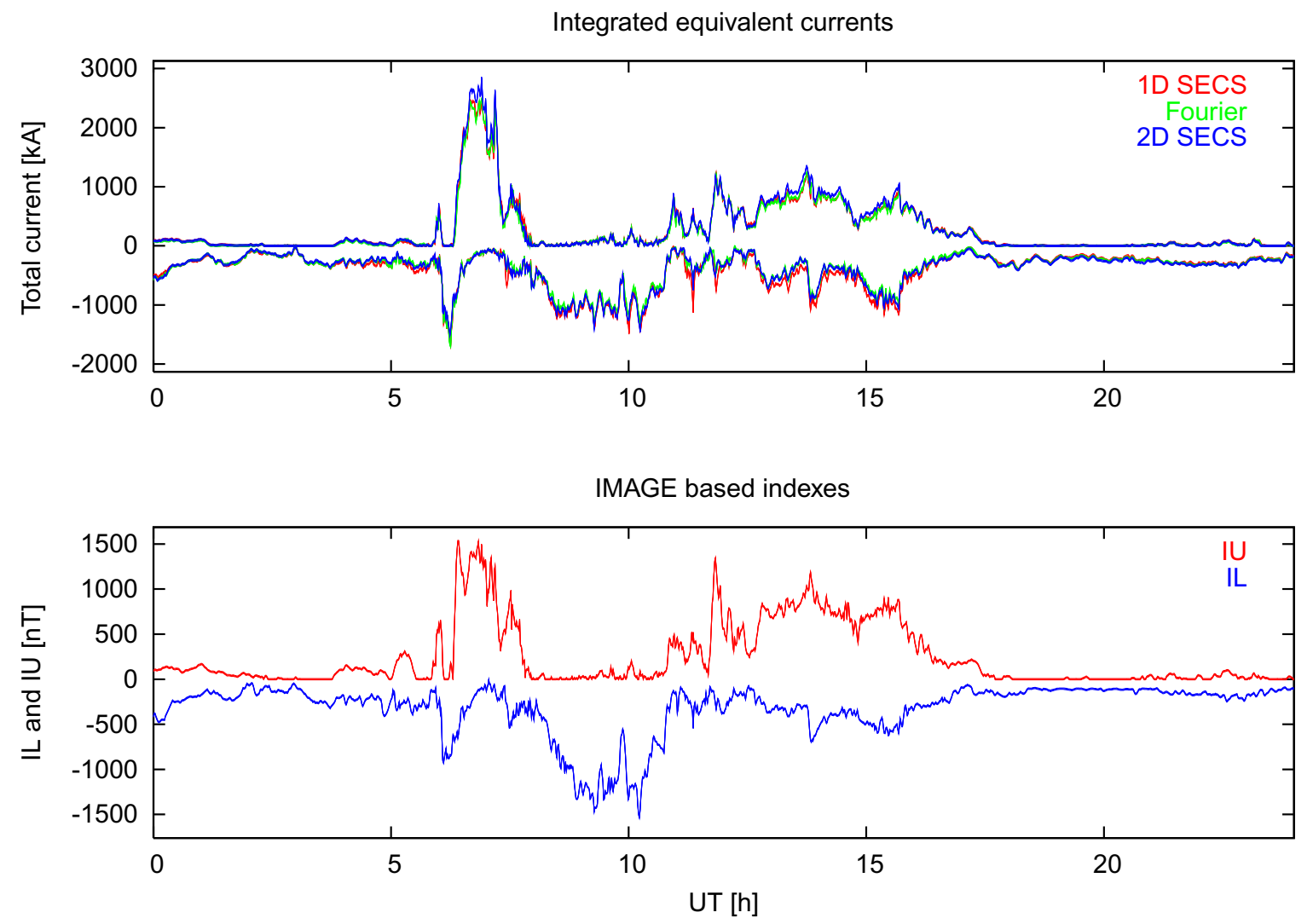

Fig. 12. Integrated eastward (+) and westward (-) currents for the whole day 24/11/2001 together with IMAGE based indices IU and IL, corresponding to $\mathrm{AU}$ and $\mathrm{AL}$, respectively. 
A $16: 42: 0$

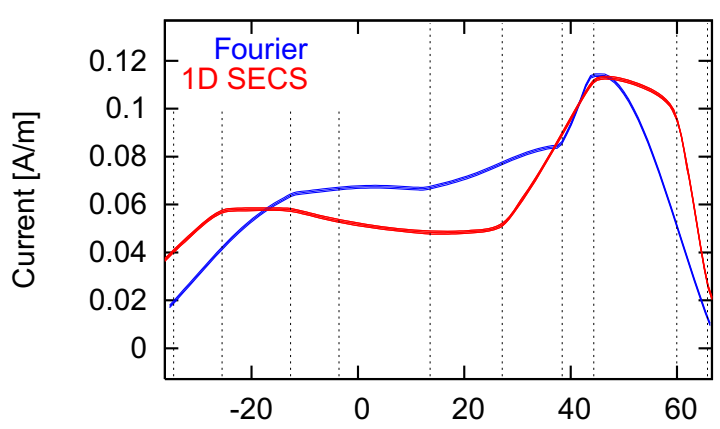

C $18: 30: 0$

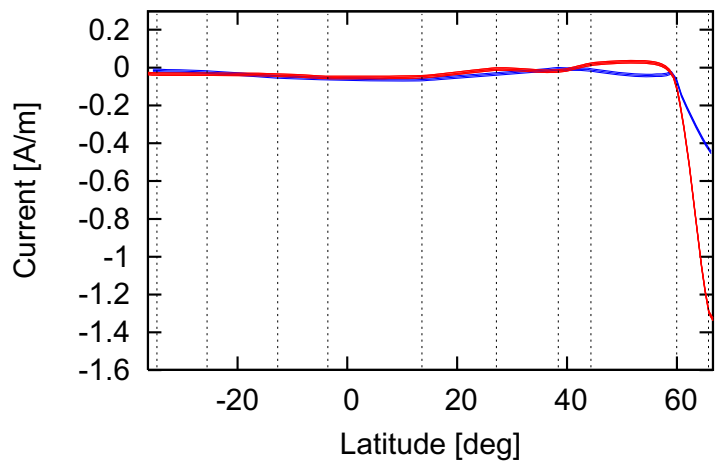

B $17: 30: 0$

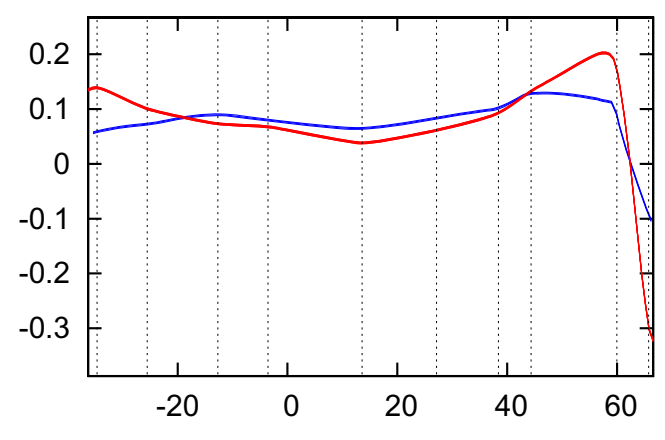

D $19: 30: 0$

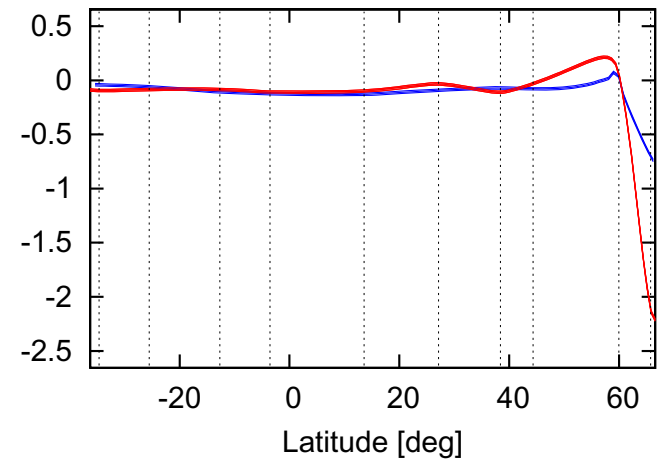

Fig. 13. Snapshots of longitudinal current distributions obtained from $210 \mathrm{MM}$ data for 6/4/2000 by 1 D SECS and Fourier methods. Eastward currents positive.

Table 2. The percentual errors of the 1D SECS and Fourier results, as compared to the 2D SECS results, for examples 1-3 (see text). Upper part: error in the current profiles averaged over latitude and timesteps (as in Eq. (11), but $J_{\text {Orig }}$ replaced with $J_{2 D}$ ). Lower part: error in the integrated currents averaged over timesteps (as in Eq. (12)).

\begin{tabular}{cccc}
\hline & Ex. 1 & Ex. 2 & Ex. 3 \\
\hline 1D SECS & 9.4 & 5.4 & 6.6 \\
\hline Fourier & 11.7 & 6.4 & 9.4 \\
\hline \hline 1D SECS & 6.3 & 5.1 & 5.6 \\
\hline Fourier & 8.6 & 3.5 & 4.9
\end{tabular}

$2 \mathrm{D}$ for the 2D SECS method. It should be remembered that the results of the 2D SECS case were used as reference when calculating the errors in Table 2 and consequently these error estimates themselves are bit uncertain. Nevertheless, it can be concluded that the 1D SECS method gives slightly more accurate current profiles, whereas the Fourier method seems to give a little better estimate for the integrated currents, except in the first example. This difference may be caused e.g. by slight oscillations in the Fourier current profiles, which average to zero in the integration. In general the errors in the integrated currents are smaller than in the actual current profiles.

In Fig. 12 we have compared the integrated currents for the whole day 24/11/2001 with IMAGE based estimates of the AL and AU indices, called IL and IU, respectively (Kauristie et al., 1996). It can be seen that the IL and IU indices give a reasonable estimate of the electrojet currents, but the agreement is not perfect. For example, the largest positive and negative values of the indices are about the same whereas the peak values of the eastward currents are larger than those of the westward currents. The integrated currents in Fig. 12 were calculated without data from BJN (see Fig. 2), as it was not available for the whole day. Nevertheless, the results of the three different methods are almost identical.

In the last example we used data from the 210 degree Magnetic Meridian chain in order to test the applicability of the 1D SECS and Fourier methods in magnetometer chains of different lengths. The 1D methods were recalibrated for this geometrically different situation with synthetic data, i.e. the spacing of the 1D SECSs, the $\epsilon$ parameter, the Fourier wavelength cut-off and the amount to extend the calculation area outside the magnetometer chain were adjusted. The synthetic data we used simulated different kins of equatorial and polar electrojets, in the same manner as in section 4 for the IMAGE network. The ionospheric equivalent currents calculated for the beginning of the April 2000 storm (6-7/4/2000) are shown in Fig. 13. Unfortunately there was no data from ADL so it was replaced by CAN (Fig. 4), which is approximately at the same latitude. The current distributions obtained using different 1D methods are similar in shape, except for the northern border of the analysis area where the 1D SECS method gives much larger westward currents. Comparison with simulated calibration cases (not shown) indicates that most of this northern electrojet is real, although current distributions that extend outside the analysis area may not be reproduced accurately. In general, 


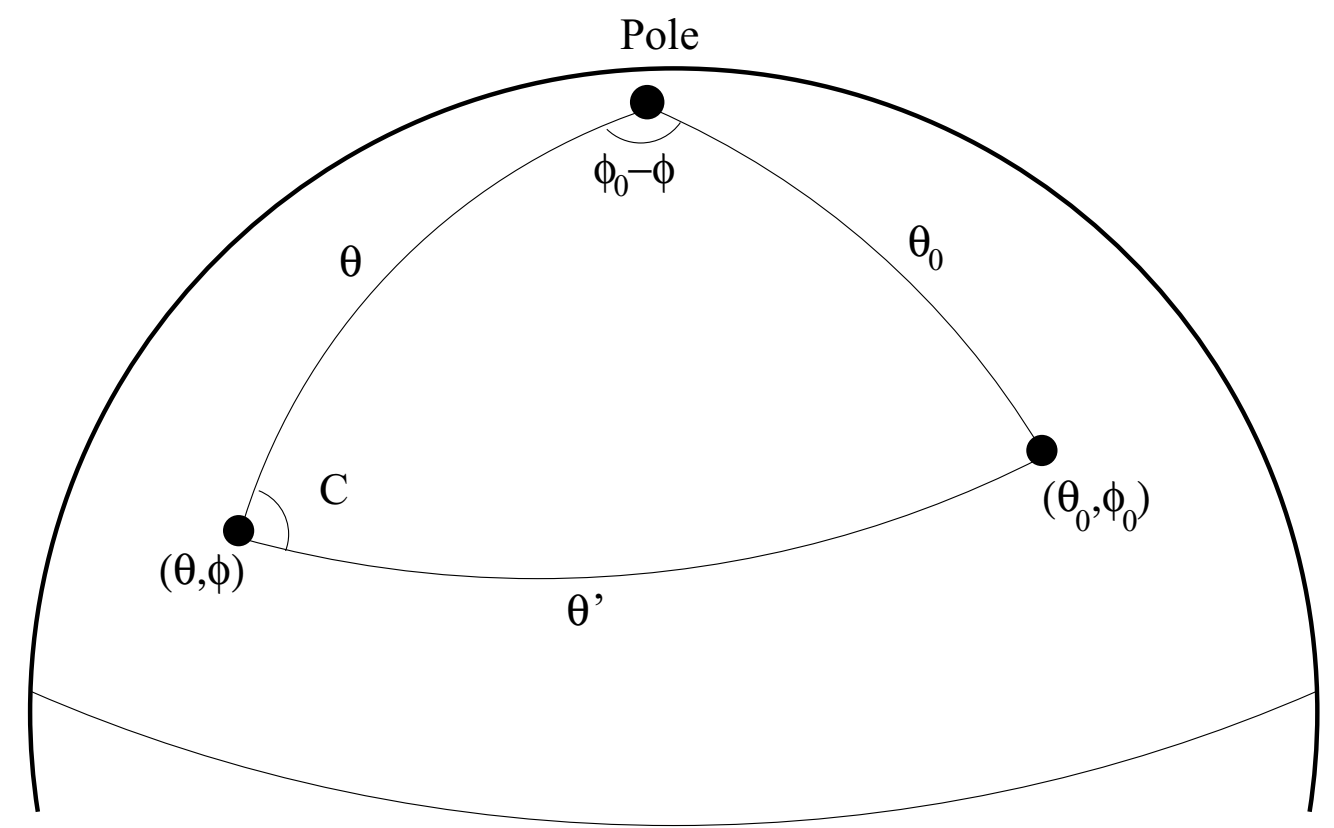

Fig. 14. Geometry of the 1D SECS current density calculation.

the 1D SECS results should be more accurate than those of the Fourier method, as expected when using data from long magnetometer chains where effects of spherical geometry are more important.

\section{Conclusions}

We have presented a new 1D method for determining ionospheric equivalent currents from ground magnetometer measurements. This 1D SECS method is applicable to electrojet situations that closely resemble the $1 \mathrm{D}$ geometry of the model and it can be used even if data along only one chain of magnetometers is available, as is the case e.g. with the Greenland magnetometer chain ${ }^{3}$ and satellite measurements. In contrast to other existing 1D upward continuation methods, e.g. Fourier expansion, the 1D SECS method respects the spherical geometry of the situation. Furthermore, there is no need to specify any kind of global wavelength cut-offs (as in harmonic expansions) and the spacing of 1D SECSs can vary with latitude. Smaller spacing (better resolution) may be used over areas where magnetometers are closer to each other.

Tests using simulated data at the IMAGE magnetometer stations indicate that the 1D SECS method indeed works well in various electrojet situations. Test cases with real data from the IMAGE chain confirm this and also indicate that the 1D SECS method gives reliable results even when the ionospheric currents are not $1 \mathrm{D}$. Especially the integrated currents given by the 1D SECS method are almost identical to the results of the more accurate 2D SECS method. As the 1D SECS method is computationally efficient (IMAGE data for a whole day is prosessed in less than one minute using a standard PC), it seems that there is no need to use local magnetic indices (e.g. IU and IL) to estimate the electrojet currents, for the currents can be directly calculated from the magnetic

http://web.dmi.dk/fsweb/projects/chain/ data with little extra effort. As a future work we will use the 1D SECS method (with slight modifications) to estimate the ionospheric currents from satellite measurements of the magnetic disturbance field.

Acknowledgments. We would like to thank M. Ichiki and another referee for their comments on the manuscript. The work of H.V. is supported by the Finnish Graduate School in Astronomy and Space Physics. The work of O.A. is supported by the Academy of Finland. The IMAGE magnetometer network is an international project coordinated by the Finnish Meteorological institute (http://www.geo.fmi.fi/image). The $210 \mathrm{MM}$ magnetic observations database is managed by the Solar-Terrestrial Environment Laboratory, Nagoya University (http://stdb2.stelab.nagoyau.ac.jp/mm210/).

\section{Appendix A.}

In this Appendix we outline the derivation of Eq. (4) for the 1D SECS current density. Our starting point is Eq. (3), in which the angle $\theta^{\prime}$ and unit vector $\hat{\mathbf{e}}_{\phi^{\prime}}$ have to be expressed in terms of coordinates $\theta, \theta_{0}, \phi_{0}$ and unit vectors $\hat{\mathbf{e}}_{\theta}, \hat{\mathbf{e}}_{\phi}$. As stated in the text the pole of the primed system is located at $\left(\theta_{0}, \phi_{0}\right)$ and the point where we want to evaluate the current density is at $(\theta, \phi)$. Because of symmetry we may set $\phi=0$. $\theta^{\prime}$ is the latitude of the point $(\theta, \phi)$ in the primed system.

According to basic trigonometry

$$
\cot \left(\frac{\theta^{\prime}}{2}\right)=\frac{\sin \theta^{\prime}}{1-\cos \theta^{\prime}} .
$$

Also, it is clear from Fig. 14 that the unit vector $\hat{\mathbf{e}}_{\phi^{\prime}}$ can be expressed as

$$
\hat{\mathbf{e}}_{\phi^{\prime}}=\hat{\mathbf{e}}_{\phi} \cos C+\hat{\mathbf{e}}_{\theta} \sin C .
$$

Using identities of spherical trigonometry it is easy to show that

$$
\sin C=\frac{\sin \theta_{0} \sin \phi_{0}}{\sin \theta^{\prime}}
$$




$$
\begin{gathered}
\cos C=\frac{\cos \theta_{0}-\cos \theta \cos \theta^{\prime}}{\sin \theta \sin \theta^{\prime}} \\
\cos \theta^{\prime}=\cos \theta \cos \theta_{0}+\sin \theta \sin \theta_{0} \cos \phi_{0} .
\end{gathered}
$$

By inserting the above expressions to the integral in Eq. (3) the $\hat{\mathrm{e}}_{\theta}$ and $\hat{\mathrm{e}}_{\phi}$ parts of the current density $\mathbf{J}_{1 D}$ may be calculated. The $\theta$ component vanishes, as can be expected because of symmetry, and the $\phi$ component results in integral

$$
2 \int_{0}^{\pi} \frac{\cos \theta_{0} \sin \theta+\cos \theta \sin \theta_{0} \cos \phi_{0}}{1-\cos \theta \cos \theta_{0}-\sin \theta \sin \theta_{0} \cos \phi_{0}} d \phi_{0}
$$

This can be calculated e.g. by using a formula (Gradshteyn and Ryzhik, 1965, p. 366, equation 3.613.1),

$$
\int_{0}^{\pi} \frac{\cos n x d x}{1+e \cos x}=\frac{\pi}{\sqrt{1-e^{2}}}\left(\frac{\sqrt{1-e^{2}}-1}{e}\right)^{n},
$$

in the special cases $n=0$ and $n=1$. The above expression is valid when $e^{2}<1$. This condition does not hold if $\theta=\theta_{0}$, at which point the current density is discontinuous. The result of the integral in Eq. (A6) can be simplified to

$$
2 \pi\left\{\begin{array}{ll}
-\tan \left(\frac{\theta}{2}\right), & \theta<\theta_{0} \\
\cot \left(\frac{\theta}{2}\right) & , \theta>\theta_{0}
\end{array},\right.
$$

which gives the result in Eq. (4).

\section{Appendix B.}

In this Appendix we outline the derivation of Eqs. (6) and (7) for the 1D SECS magnetic field components. Our starting point is Eq. (5) for the vector potential. We are again allowed to choose the observation point $(r, \theta, \phi)$ so that $\phi=0$. In this case the unit vector $\hat{\mathrm{e}}_{\phi_{1}}$ can be written in Cartesian coordinate system as

$$
\hat{\mathrm{e}}_{\phi_{1}}=-\sin \phi_{1} \hat{\mathrm{e}}_{x}+\cos \phi_{1} \hat{\mathrm{e}}_{y} .
$$

Integration over $r_{1}$ is trivial because of the $\delta$-function in $\mathbf{J}_{1 D}$. The remaining double integral is

$$
\begin{aligned}
\mathbf{A}= & \frac{\mu_{0} I_{0} R_{I}}{8 \pi} \int_{0}^{\pi}\left[\cos \theta_{1}-\operatorname{sign}\left(\cos \theta_{1}-\cos \theta_{0}\right)\right] * \\
& * \int_{0}^{2 \pi} \frac{-\sin \phi_{1} \hat{\mathrm{e}}_{x}+\cos \phi_{1} \hat{\mathrm{e}}_{y}}{\left|\mathbf{r}-\mathbf{r}_{1}\right|} d \phi_{1} d \theta_{1} .
\end{aligned}
$$

Clearly, the $x$ component vanishes in the $\phi_{1}$ integration so that $\mathbf{A}$ is directed along the $\hat{\mathrm{e}}_{y}=\hat{\mathrm{e}}_{\phi}$ unit vector. The remaining $\phi_{1}$ integral may be calculated by expanding the denominator in spherical harmonics,

$$
\begin{aligned}
\frac{1}{\left|\mathbf{r}-\mathbf{r}_{1}\right|}= & 4 \pi \sum_{l=0}^{\infty} \sum_{m=-l}^{l} \frac{1}{2 l+1} * \\
& * \frac{r_{<}^{l}}{r_{>}^{l+1}} Y_{l, m}^{*}\left(\theta_{1}, \phi_{1}\right) Y_{l, m}(\theta, \phi) .
\end{aligned}
$$

Here we have defined $r_{<}=\min \left(r, R_{I}\right), r_{>}=\max \left(r, R_{I}\right)$. Because $Y_{l, m}^{*}\left(\theta_{1}, \phi_{1}\right) \propto e^{-i m \phi_{1}}$ and $\cos \phi_{1}=\operatorname{Re}\left(e^{i \phi_{1}}\right)$ the only value of $m$ that does produce nonzero integral is $m=1$.

The remaining task is to calculate the $\theta_{1}$ integral

$$
\int_{0}^{\pi}\left[\cos \theta_{1}-\operatorname{sign}\left(\cos \theta_{1}-\cos \theta_{0}\right)\right] P_{l}^{1}\left(\cos \theta_{1}\right) d \theta_{1} \text {. }
$$

Here we have used the Condon-Shortley phase convention in the definition of the associated Legendre polynomials $P_{l}^{1}$. Using their properties and integrating by parts yields the result of the above integral as $2 \delta_{l, 0}-2 P_{l}\left(\cos \theta_{0}\right)$.

Using the above results and writing the remaining spherical harmonics in terms of Legendre polynomials the vector potential can be expressed as

$$
\begin{gathered}
\mathbf{A}(\mathbf{r})=\frac{-\mu_{0} I_{0} R_{I}}{2 r_{>}} \sum_{l=1}^{\infty}\left(\frac{r_{<}}{r_{>}}\right)^{l} \frac{1}{l(l+1)} * \\
* P_{l}\left(\cos \theta_{0}\right) P_{l}^{1}(\cos \theta) \hat{\mathrm{e}}_{\phi} .
\end{gathered}
$$

The magnetic field can be calculated as $\mathbf{B}=\nabla \times \mathbf{A}$. The azimuthal component $B_{\phi}$ of the field is zero and in the case $r<R_{I}$ the other components are given in Eqs. (6) and (7). In the case $r>R_{I}$ the $r$ - and $\theta$-components are

$$
\begin{gathered}
B_{r}=\frac{\mu_{0} I_{0} R_{I}}{2 r^{2}} \sum_{l=1}^{\infty}\left(\frac{R_{I}}{r}\right)^{l} P_{l}\left(\cos \theta_{0}\right) P_{l}(\cos \theta) \\
B_{\theta}=\frac{-\mu_{0} I_{0} R_{I}}{2 r^{2}} \sum_{l=1}^{\infty}\left(\frac{R_{I}}{r}\right)^{l} \frac{1}{l+1} * \\
* P_{l}\left(\cos \theta_{0}\right) P_{l}^{1}(\cos \theta) .
\end{gathered}
$$

\section{References}

Amm, O., Ionospheric elementary current systems in spherical coordinates and their application, J. Geomag. Geoelectr., 49, 947-955, 1997.

Amm, O. and A. Viljanen, Ionospheric disturbance magnetic field continuation from the ground to the ionosphere using spherical elementary current systems, Earth Planets Space, 51, 431-440, 1999.

Fukushima, N., Generalized theorem for no ground magnetic effect of vertical currents connected with Pedersen currents in the uniformconductivity ionosphere, Rep. Ionos. Space. Res. Japan, 30, 35-40, 1976.

Gradshteyn, I. and I. Ryzhik, Table of Integrals, Series and Products, 4th ed., 1086 pp, Academic Press, New York, 1965.

Haines, G., Spherical Cap Harmonic Analysis, J. Geophys. Res., 90(B3), 2583-2591, 1985.

Kauristie, K., T. Pulkkinen, R. Pellinen, and H. Opgenoorth, What can we tell about global auroral-electrojet activity from a single meridional magnetometer chain?, Ann. Geophys., 14, 1177-1185, 1996.

Mersmann, U., W. Baumjohann, F. Küppers, and K. Lange, Analysis of an eastward electrojet by means of upward continuation of ground-based magnetometer data, J. Geophys., 45, 281-298, 1979.

Popov, V., V. Papitashvili, and J. Watermann, Modeling of equivalent ionospheric currents from meridian magnetometer chain data, Earth Planets Space, 53, 129-137, 2001.

Press, W., S. Teukolsky, W. Vetterling, and B. Flannery, Numerical Recipes in Fortran 77. The Art of Scientific Computing, 2nd ed., 973 pp, Cambridge Univ. Press, Cambridge, 1992.

Pulkkinen, A., O. Amm, A. Viljanen, and BEAR Working Group, Ionospheric equivalent current distributions determined with the method of spherical elementary current systems, J. Geophys. Res., 108(A2), 1053, doi:10.1029/2001JA005085, 2003a.

Pulkkinen, A., O. Amm, A. Viljanen, and BEAR Working Group, Separation of the geomagnetic variation field on the ground into external and internal parts using the spherical elementary current system method, Earth Planets Space, 55, 117-129, 2003 b.

Untiedt, J. and W. Baumjohann, Studies of polar current systems using the IMS Scandinavian magnetometer array, Space Sci. Rev., 63, 245-390, 1993.

H. Vanhamäki (e-mail: heikki.vanhamaki@fmi.fi), O. Amm, and A. Viljanen 